\title{
Basis Optimization Renormalization Group for Quantum Hamiltonian
}

\author{
Takanori Sugihara* \\ Department of Physics, Nagoya University, \\ Chikusa, Nagoya 464-8602, Japan
}

We find an algorithm of numerical renormalization group for spin chain models. The essence of this algorithm is orthogonal transformation of basis states, which is useful for reducing the number of relevant basis states to create effective Hamiltonian. We define two types of rotations and combine them to create appropriate orthogonal transformation.

\section{INTRODUCTION}

A method of Hamiltonian diagonalization is suitable for calculating physical quantities associated with wavefunctions, such as structure functions and form factors. However, we cannot diagonalize Hamiltonian directly in quantum field theories because dimension of Hilbert space is generally infinite. To create effective Hamiltonian, we extend a technique of NRG (numerical renormalization group) proposed by K. Wilson [1].

In spin chain models, any state can be expanded with a direct product of two sets of basis states, each of which is for one of two spin blocks. For a finite lattice, we can calculate wavefunction $\Psi_{i j}$ of the ground state by diagonalizing Hamiltonian. In Ref. [2], S.White has found that singular values $D_{k}$ of target-state wavefunction $\Psi_{i j}=\sum_{k} U_{i k} D_{k} V_{k j}$ are useful for the purpose of creating effective Hamiltonian.

$|\Psi\rangle=\sum_{i} \sum_{j} \Psi_{i j}|i\rangle|j\rangle=\sum_{k} D_{k}\left|u_{k}\right\rangle\left|v_{k}\right\rangle$,

where $\left|u_{k}\right\rangle \equiv \sum_{i} U_{i k}|i\rangle$ and $\left|v_{k}\right\rangle \equiv \sum_{j} V_{k j}|j\rangle$. If $\left|D_{k}\right|$ is large, the corresponding basis state is relevant. However, if $\left|D_{k}\right|$ is small, the corresponding basis state is not relevant and can be thrown away from calculation. We can control calculation accuracy using singular values $D_{k}$ when we truncate Hilbert space. Magnitude of $\left|D_{k}\right|$ determines the relevance of basis states in the target state. Basis truncation based on singular values

*Email: sugihara@eken.phys.nagoya-u.ac.jp works well in one-dimensional spin models such as Heisenberg and Hubbard models 23. This is an extension of Wilson's NRG and called DMRG (density matrix renormalization group). White has shown that there exists orthogonal transformation that largely reduces the number of basis states with high calculation accuracy. However, we cannot assure that a number of singular values take values of nearly zero in general cases. In order to create optimized basis states without relying on density matrix, we will find another useful basis transformation. Our aim is to find basis transformation that make coefficients (i.e. transformed wavefunctions) exactly zero when target states are expanded with new basis states.

\section{FORMULATION}

Let us consider $S=1 / 2$ Heisenberg chain. Since we cannot diagonalize infinite dimensional Hamiltonian directly, we start from a finite lattice. We extend a lattice step by step by adding one site to it in each RG transformation. $\left|u_{i}\right\rangle$ is a complete set of basis states for a finite lattice $L$. Hilbert space of an extended lattice with size $(L+1)$ is spanned with a set of basis states $\left|u_{i}\right\rangle|j\rangle$, where $|j\rangle$ is an eigenstate of $z$-component of a spin operator for the $(L+1)$-th site

$\hat{S}^{z}|j\rangle= \pm \frac{1}{2}|j\rangle \quad$ for $\quad j= \pm$. 
We want to find a rotation matrix $U$ that decrease the number of relevant basis states.

$\left|u_{j}^{\prime}\right\rangle=\sum_{i} U_{i j}\left|u_{i}\right\rangle$.

In general, any orthogonal matrix can be expressed as a product of two-dimensional rotation matrices $R$.

$R_{(k, l)}(\theta)=k\left(\begin{array}{ccccc} & k & & l & \\ & \vdots & & \vdots & \\ \ldots & \cos \theta & \ldots & -\sin \theta & \ldots \\ & \vdots & \ddots & \vdots & \\ \ldots & \sin \theta & \ldots & \cos \theta & \ldots \\ \vdots & & \vdots & \end{array}\right)$

This matrix rotates the $k$-th and $l$-th basis states by an angle $\theta$. Let us consider a ground state that is an eigenstate of a Hamiltonian.

$|\Psi\rangle=\sum_{i j} \Psi_{i j}\left|u_{i}\right\rangle|j\rangle$.

We consider a part of this target state.

$$
\begin{aligned}
|\Psi\rangle_{(1,2)} & =\Psi_{1-}\left|u_{1}\right\rangle|-\rangle+\Psi_{1+}\left|u_{1}\right\rangle|+\rangle \\
& +\Psi_{2-}\left|u_{2}\right\rangle|-\rangle+\Psi_{2+}\left|u_{2}\right\rangle|+\rangle .
\end{aligned}
$$

If we rotate two basis states $\left|u_{1}\right\rangle$ and $\left|u_{2}\right\rangle$

$$
\begin{aligned}
\left|u_{1}\right\rangle & =\cos \theta\left|u_{1}^{\prime}\right\rangle-\sin \theta\left|u_{2}^{\prime}\right\rangle, \\
\left|u_{2}\right\rangle & =\sin \theta\left|u_{1}^{\prime}\right\rangle+\cos \theta\left|u_{2}^{\prime}\right\rangle,
\end{aligned}
$$

we have

$$
\begin{aligned}
|\Psi\rangle_{(1,2)} & =\Psi_{1-}^{\prime}\left|u_{1}^{\prime}\right\rangle|-\rangle+\Psi_{1+}^{\prime}\left|u_{1}^{\prime}\right\rangle|+\rangle \\
& +\Psi_{2-}^{\prime}\left|u_{2}^{\prime}\right\rangle|-\rangle+\Psi_{2+}^{\prime}\left|u_{2}^{\prime}\right\rangle|+\rangle,
\end{aligned}
$$

where $\Psi^{\prime}$ are transformed wavefunctions

$$
\begin{aligned}
& \Psi_{1-}^{\prime}=\Psi_{1-} \cos \theta+\Psi_{2-} \sin \theta, \\
& \Psi_{1+}^{\prime}=\Psi_{1+} \cos \theta+\Psi_{2+} \sin \theta, \\
& \Psi_{2-}^{\prime}=-\Psi_{1-} \sin \theta+\Psi_{2-} \cos \theta, \\
& \Psi_{2+}^{\prime}=-\Psi_{1+} \sin \theta+\Psi_{2+} \cos \theta .
\end{aligned}
$$

We define the following two types of rotations. Rotation I is defined to make the fourth component zero $\Psi_{2+}^{\prime}=0$;

$$
\left(\begin{array}{l}
\Psi_{1-} \\
\Psi_{1+} \\
\Psi_{2-} \\
\Psi_{2+}
\end{array}\right) \stackrel{\mathrm{I}}{\rightarrow}\left(\begin{array}{c}
\Psi_{1-}^{\prime} \\
\Psi_{1+}^{\prime} \\
\Psi_{2-}^{\prime} \\
0
\end{array}\right)
$$

A condition $\Psi_{2+}^{\prime}=0$ determines the value of $\theta$. Rotation II is defined to make the third component zero $\Psi_{2-}=0$ when components $\Psi_{1+}$ and $\Psi_{2+}$ are zero;

$\left(\begin{array}{c}\Psi_{1-} \\ 0 \\ \Psi_{2-} \\ 0\end{array}\right) \stackrel{\text { II }}{\rightarrow}\left(\begin{array}{c}\Psi_{1-}^{\prime} \\ 0 \\ 0 \\ 0\end{array}\right)$

When $\Psi_{1+}=\Psi_{2+}=0$, the transformed components $\Psi_{1+}^{\prime}$ and $\Psi_{2+}^{\prime}$ are always zero. Therefore, we can make $\Psi_{2-}^{\prime}$ zero by choosing $\theta$ appropriately.

In order to understand how these two rotations are used to find relevant basis states, let us consider a simple example where a target state is expanded with six basis states.

$|\Psi\rangle=\sum_{i=1}^{3} \sum_{j= \pm} \Psi_{i j}\left|u_{i}\right\rangle|j\rangle$.

The following is transformation of the sixdimensional vector of the target-state wavefunction $\Psi_{i j}$.

$$
\left(\begin{array}{l}
\Psi_{1-} \\
\Psi_{1+} \\
\Psi_{2-} \\
\Psi_{2+} \\
\Psi_{3-} \\
\Psi_{3+}
\end{array}\right) \stackrel{\mathrm{I}}{\rightarrow}\left(\begin{array}{c}
\Psi_{1-}^{\prime} \\
\Psi_{1+}^{\prime} \\
\Psi_{2-}^{\prime} \\
0 \\
\Psi_{3-} \\
\Psi_{3+}
\end{array}\right) \stackrel{\mathrm{I}}{\rightarrow}\left(\begin{array}{c}
\Psi_{1-}^{\prime \prime} \\
\Psi_{1+}^{\prime \prime} \\
\Psi_{2-}^{\prime} \\
0 \\
\Psi_{3-}^{\prime} \\
0
\end{array}\right) \stackrel{\text { II }}{\rightarrow}\left(\begin{array}{c}
\Psi_{1-}^{\prime \prime} \\
\Psi_{1+}^{\prime \prime} \\
\Psi_{2-}^{\prime \prime} \\
0 \\
0 \\
0
\end{array}\right)
$$

We obtain the final form by transforming the vector with two rotations appropriately. If a transformed wavefunction is zero, the corresponding basis state is irrelevant and can be neglected. That is, only the first three basis states $\left(\left|u_{1}^{\prime \prime}\right\rangle| \pm\rangle,\left|u_{2}^{\prime \prime}\right\rangle|-\rangle\right)$ are relevant and others $\left(\left|u_{2}^{\prime \prime}\right\rangle|+\rangle,\left|u_{3}^{\prime \prime}\right\rangle| \pm\rangle\right)$ are redundant. The former three relevant basis states can reproduce the target state exactly without other basis states. There exists orthogonal transformation that reduces the number of relevant basis states into three without causing calculation error. This is also true for arbitrary dimensions other than six of this example.

Let us consider a case where we target ground and first-excited states. The followings are wave- 
function vectors of the target states.

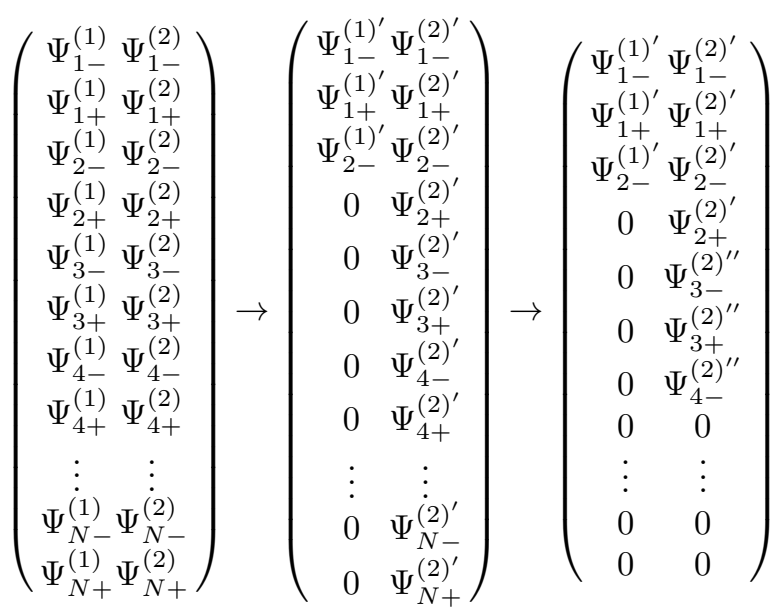

The indices (1) and (2) indicate the ground and first-excited states, respectively. We optimize basis states in two steps. In the first step, we target the ground state $\Psi^{(1)}$ and do not take care of the first excited state $\Psi^{(2)}$. In the same way as the previous example (4), we can find three relevant basis states that can reproduce the ground state exactly by using a combination of rotations I and II. In the second step, we target the first excited state $\Psi^{(2)}$ to optimize basis states further. When we rotate basis states, we don't touch the first four basis states $\left(\left|u_{1}^{\prime}\right\rangle| \pm\rangle,\left|u_{2}^{\prime}\right\rangle| \pm\rangle\right)$. We apply a combination of rotations I and II to other $(2 N-4)$ pieces of basis states $\left(\left|u_{3}^{\prime}\right\rangle| \pm\rangle,\left|u_{4}^{\prime}\right\rangle| \pm\rangle\right.$, ..., $\left.\left|u_{N}^{\prime}\right\rangle| \pm\rangle\right)$. Finally, we obtain seven basis states $\left(\left|u_{1}^{\prime \prime}\right\rangle| \pm\rangle,\left|u_{2}^{\prime \prime}\right\rangle| \pm\rangle,\left|u_{3}^{\prime \prime}\right\rangle| \pm\rangle,\left|u_{4}^{\prime \prime}\right\rangle|-\rangle\right)$ that are relevant for reproducing the ground and first excited states exactly. In the same way, we can also create relevant basis states when arbitrary number of eigenstates are targeted. The number of target states can be chosen according to the desired calculation accuracy. We can use this technique of basis transformation to truncate Hilbert space and create effective Hamiltonian in NRG. Our NRG algorithm for $S=1 / 2$ Heisenberg chain is as follows:

1. Diagonalize $H_{L}\left(H_{L} \boldsymbol{u}_{n}=E_{n} \boldsymbol{u}_{n}\right)$, which is Hamiltonian for a finite lattice $L$.

2. Transform basis states $\boldsymbol{u}_{n} \rightarrow \boldsymbol{u}_{n}^{\prime}$ using type I and II rotations to make wavefunctions of target states zero as many as possible.
3. Create effective Hamiltonian $\bar{H}_{L}=$ $O_{L}^{\dagger} H_{L} O_{L}$, where $O_{L}=\left(\boldsymbol{u}_{1}^{\prime}, \boldsymbol{u}_{2}^{\prime}, \ldots, \boldsymbol{u}_{M}^{\prime}\right)$. Dimension of $\bar{H}_{L}$ is $M$.

4. Add one site to $\bar{H}_{L}$ to provide next Hamiltonian $H_{L+1}$. Dimension of $H_{L+1}$ is $2 M$.

5. Regard $H_{L+1}$ as $H_{L}$, and go to step 1 .

These steps are iterated till vacuum energy per site converges with the desired accuracy. I have applied this algorithm to $S=1 / 2$ Heisenberg chain and calculated vacuum energy per site. An exact value of vacuum energy has been obtained using Bethe ansatz. The difference between our result and exact value is about $3.9 \times 10^{-4}$ for parameters $M=256$ and $L=2000$.

\section{CONCLUSION}

We have found basis transformation that largely reduces the number of relevant basis states. Especially, three states are sufficient for reproducing one target state in each RG step. Relevant basis states reproduce target states exactly in each RG step. Therefore, if dimension of effective Hamiltonian $M$ is sufficiently large, calculation errors come from non-targeted states. Future problems are improvement of accuracy, calculation of correlation functions, calculation of Haldane gap, and application to other models.

\section{REFERENCES}

1. K. G. Wilson, Rev. Mod. Phys. 47, 773 (1975).

2. S. R. White, Phys. Rev. Lett. 69, 2863 (1992), Phys. Rev. B 48, 10345 (1993), Phys. Rep. 301, 187 (1998).

3. S. Noack and S. R. White, in Density Matrix Renormalization, Lecture Notes in Physics, edited by I. Peschel et al. (Springer-Verlag, New York, 1999). 